\title{
Hiperglicemia Pós-Prandial em Pacientes com Diabetes Melito Tipo 2
}

\section{artigo original}

\author{
ANDRÉ LUIZ CRUZES \\ Cláudia Elaine T. Bosco \\ ELAINE V. PANDINI \\ Miguel A. M. HeRnANDEZ \\ REgINA C. Q. DA SILVA
}

Departamentos de Endocrinologia (ALC, MAMH, RCQS), Enfermagem (CETB) e Nutrição (EVP) da Associação de Diabetes Juvenil (ADJ) da Região Noroeste do Estado de São Paulo, Birigui, SP, Brasil.

\section{RESUMO}

Objetivos: Por meio da análise de um grupo de pacientes com DM2, temos por objetivos: avaliar por monitoração da glicose subcutânea (MGSG) as excursões pós-prandiais da glicose subcutânea; analisar as correlações da glicose subcutânea e glicemias capilares com a HbA1c e avaliar a eficácia, a segurança e a tolerabilidade da MGSC. Métodos: Foram analisados 40 pacientes com DM2, com $\mathrm{HbA} 1 \mathrm{c}$ até $7,3 \%$, em uso de agentes orais e com menos de 10 anos de diagnóstico. Foram submetidos à MGSC por 72 horas, cujos dados foram confrontados com a HbA1c e as glicemias capilares. Resultados: A excursão média da glicose subcutânea do desjejum foi estatisticamente maior que a do jantar (95\%IC -24,96 a -1,66). A razão excursão da glicose subcutânea/conteúdo de carboidrato da refeição foi estatisticamente maior no desjejum que nas demais refeições $(p=0,001)$. Não houve correlação entre as taxas de glicose subcutânea e as glicemias capilares com HbA1c. Não houve complicações com o uso da MGSC. Conclusões: As excursões de glicose subcutânea predominaram no desjejum. Não se encontrou correlação entre $\mathrm{HbA1c}$ e parâmetros de controle glicêmico. A MGSC mostrou-se eficaz, segura e bem tolerada nesse grupo de pacientes. (Arq Bras Endocrinol Metab 2008; 52/4:642-648)

Descritores: Hiperglicemia pós-prandial; Glicemia pós-desjejum; Monitoração da glicose subcutânea; Índice glicêmico; Hemoglobina glicada; Diabetes melito tipo 2.

\section{ABSTRACT}

Post-Prandial Hyperglycemia in Type 2 Diabetes Patients.

Objectives: Through the analysis of a group of type 2 diabetes patients, the aims of this study are: to evaluate through CGMS (continuous glucose monitoring system) the post-prandial subcutaneous glucose excursions; to analyze the correlations between subcutaneous glucose and capillary glycemia with $\mathrm{HbA} 1 \mathrm{c}$ and to evaluate the effectiveness, safety and broadmindedness of the CGMS. Methods: Forty type 2 diabetes patients were analyzed, with $\mathrm{HbA} 1 \mathrm{c}$ until $7.3 \%$, in use of oral agents and within less than a ten-year diagnosis. They were submitted to CGMS for $72 \mathrm{~h}$, whose data were compared with $\mathrm{HbA} 1 \mathrm{c}$ and capillary glycemia. Results: The average subcutaneous glucose excursion at breakfast was statistically bigger than at dinner $(95 \% \mathrm{Cl}-24,96$ a $-1,66)$. The subcutaneous glucose excursion/meal carbohydrates content ratio was statistically bigger at breakfast than at other meals $(p 0,001)$. There was no correlation between the levels of subcutaneous glucose and capillary glycemia with $\mathrm{HbA} 1 \mathrm{c}$. There weren't complications using CGMS. Conclusions: The subcutaneous glucose excursions were predominant at breakfast. There were no correlations between $\mathrm{HbA} 1 \mathrm{c}$ and glycemic control parameters. CGMS showed itself efficient, secure and well tolerated in this group of patients. (Arq Bras Endocrinol Metab 2008; 52/4:642-648)

Keywords: Post-prandial hyperglycemia; Post-breakfast glycemia; Continuous glucose monitoring system; Glycemic index; Glycated hemoglobin; Type 2 diabetes mellitus. 


\section{INTRODUÇÃO}

BUSCA DO CONTROLE GLICÊMICO ideal continua sendo um dos grandes desafios da terapêutica do diabetes melito, tanto tipo 1 (DMl) quanto tipo 2 (DM2). Este melhor controle comprovadamente reduz as complicações crônicas, como foi visto nos trabalhos clássicos: Diabetes control and complications trial (DCCT) e o United Kingdom prospective study (UKPDS), que mostraram redução significativa das complicações crônicas com o tratamento intensivo $(1,2)$. Os níveis de hemoglobina glicada $(\mathrm{HbAlc})<7 \%$ como referência de bom controle glicêmico têm sido questionados recentemente por publicações que sugerem metas mais rigorosas $(3,4)$. No entanto, mesmo com a adoção da automonitorização diária da glicemia capilar, o alcance da euglicemia permanece distante para a maioria dos pacientes.

O fenômeno da hiperglicemia pós-prandial é reconhecido como importante fator de risco para complicações, notadamente cardiovasculares. Vários estudos prospectivos demonstram o papel maior da glicemia pós-prandial ou da glicemia 2 horas após sobrecarga (em relação ao jejum), no risco para mortalidade e doenças cardiovasculares (DCVs) (5-10). Isso ocorre precocemente, desde a fase de tolerância diminuída à glicose, pela perda da fase rápida de secreção de insulina (10). A hiperglicemia pós-prandial também é acompanhada de uma série de outros distúrbios metabólicos, como a elevação dos triglicérides, que contribuem para a intensificação do processo aterosclerótico (11-13).

Evidências clínicas em indivíduos com DMl e DM2 sugerem que a utilização de dados da monitoração da glicose subcutânea (MGSC) possa ser útil nos ajustes terapêuticos com a finalidade de atingir metas glicêmicas mais estritas (14). A MGSC é ferramenta relativamente recente para avaliação do controle glicêmico, acompanhando de maneira ininterrupta as variações das taxas glicêmicas $(15,16)$. No momento, já há vários estudos, principalmente em DMl, mostrando segurança e boa sensibilidade para detecção de picos hiperglicêmicos pós-prandiais $(13,15-17)$. Há relatos que a glicemia pós-desjejum seja a que sofra maior variação durante o dia (18). A experiência mundial da MGSC em DM2 ainda é limitada. No Brasil, não há relato de estudos que envolvam essa tecnologia em DM2.

O objetivo deste estudo foi analisar um grupo de pacientes com DM2 bem controlados para: avaliar, por meio de MGSC, as excursões pós-prandiais da glicose subcutânea e a relação entre as excursões e o conteúdo de carboidrato em cada refeição; analisar a existência de possíveis correlações entre glicose subcutânea e HbAlc e entre glicemias capilares e HbAlc; avaliar a eficácia, a segurança e a tolerabilidade da MGSC.

\section{PACIENTES E MÉTODOS}

Estudaram-se prospectivamente, 50 pacientes com DM2 em uso de agentes orais: sulfoniluréias, metformina e tiazolidinedionas (TZD), em monoterapia ou combinados, com menos de dez anos de diagnóstico. Após a aprovação do comitê de ética da nossa instituição (Processo n ${ }^{\circ} 02 / 2006$ ), os participantes assinaram termo de consentimento.

Foram critérios de exclusão: idade superior a 65 anos, presença de nefropatia instalada (proteinúria $>300 \mathrm{mg} / 24$ horas), retinopatia, diagnóstico prévio de coronariopatia, uso de agentes anticoagulantes, gestação, uso de corticóides e/ou presença de endocrinopatias hiperglicemiantes.

Os pacientes foram submetidos à MGSC (Medtro nic ${ }^{\circledR}$-CGMS) por 72 horas, após calibração do sensor, conforme normas do fabricante. Durante o período de análise, realizaram glicemias capilares (Optium xceed ${ }^{\circledR}$, Abbott) nos seguintes horários: jejum (GCD), 2 horas após desjejum (GCPD), 2 horas após almoço (GCPA) e 2 horas após jantar (GCPJ), cujos valores foram utilizados para calibração do sensor (no total de 12 vezes durante as 72 horas de análise). $\mathrm{O}$ sensor foi instalado na região abdominal.

Os pacientes foram orientados a seguir a dieta habitual, evitando a alimentação em intervalos inferiores a 2 horas após as refeições principais. Solicitou-se recordatório alimentar minucioso durante as 72 horas de análise. Por meio desses relatórios foi realizada avaliação do conteúdo de carboidratos e do índice glicêmico (IG) das refeições. O cálculo do IG foi fundamentado na tabela internacional de valores de índice glicêmico e carga glicêmica (2002) (19).

$\mathrm{A} \mathrm{HbAlc} \mathrm{foi} \mathrm{coletada} \mathrm{no} \mathrm{dia} \mathrm{seguinte} \mathrm{à} \mathrm{retirada} \mathrm{do}$ sensor. A análise foi realizada pelo método de cromatografia líquida de alta performance (HPLC), com faixa normal: $4 \%$ a $6 \%$.

Dos 50 pacientes recrutados, 10 foram excluídos: seis por controle glicêmico insatisfatório $(\mathrm{HbAlc}>7,3)$, três por prejuízo da análise dos dados do sensor e um paciente apresentou concomitantemente os dois motivos citados.

Entre os 40 pacientes aptos para análise, houve pequenas interrupções e/ou falhas do funcionamento do sensor em cinco pacientes, mas que não foram suficientes para prejudicar a análise dos dados. As características clínicas dos pacientes estudados estão contidas na Tabela 1. 
Tabela 1. Características clínicas e terapêuticas dos indivíduos com diabetes melito do tipo 2 estudados.

\begin{tabular}{|c|c|}
\hline $\mathbf{N}$ & 40 \\
\hline $\operatorname{Sexo}(M / F)$ & $19 / 21$ \\
\hline Idade (anos) & $53,6 \pm 7,2$ \\
\hline $\mathrm{IMC}\left(\mathrm{kg} / \mathrm{m}^{2}\right)$ & $27,6 \pm 4,3$ \\
\hline Duração do diabetes (anos) & $3,8 \pm 3,3$ \\
\hline \multicolumn{2}{|l|}{ Tratamento } \\
\hline Metformina isolada & $25(62,5 \%)$ \\
\hline Sulfoniluréia isolada & $1(2,5 \%)$ \\
\hline Combinação $(M+S)$ & $12(30 \%)$ \\
\hline Metformina + TZD & $1(2,5 \%)$ \\
\hline Combinação (M + S + TZD) & $1(2,5 \%)$ \\
\hline Hipertensão arterial & $28(70 \%)$ \\
\hline Dislipidemia & $23(57,5 \%)$ \\
\hline Complicações crônicas & $6(15 \%)$ \\
\hline Neuropatia periférica' & $4(10 \%)$ \\
\hline Microproteinúria² & $1(2,5 \%)$ \\
\hline Arteriopatia periférica ${ }^{3}$ & $1(2,5 \%)$ \\
\hline
\end{tabular}

' Neuropatia periférica foi definida como alteração de sensibilidade plantar ao teste com monofilamento; ${ }^{2}$ Microproteinúria foi definida como perda de albumina urinária entre 30 a $300 \mathrm{mg} / 24$ horas; ${ }^{3}$ Arteriopatia periférica foi definida como diminuição ou ausência dos pulsos arteriais distais (pedioso e tibial posterior) à palpação.
A excursão da glicose subcutânea em cada refeição foi definida como a variação entre o valor prévio à refeição e o seu pico pós-prandial detectado pelo sensor.

Para analisar a correlação entre HbAlc e parâmetros de controle glicêmico (glicemias capilares e glicose subcutânea), foram calculados os coeficientes de correlação de Pearson (r) e os coeficientes de determinação $\left(\mathrm{R}^{2}\right)$. Para avaliar a hipótese de que havia diferença nas excursões da glicose subcutânea das refeições, foi utilizado o teste de análise de variâncias (ANOVA). Para efeito de comparação entre as refeições e o estudo da relação excursão da glicose subcutânea/conteúdo de carboidratos, foi utilizado o teste de Tukey. Consideramos significância estatística o valor de $\mathrm{p}<0,05$.

\section{RESULTADOS}

\section{Análise das excursões da glicose subcutânea}

As excursões médias da glicose subcutânea após cada refeição foram diferentes entre si (desjejum $=65,81 \pm$ $36,02 \mathrm{mg} / \mathrm{dl} ;$ almoço $=57,27 \pm 40,29 \mathrm{mg} / \mathrm{dl} \mathrm{e} \mathrm{jantar}$ $=52,5 \pm 38,27 \mathrm{mg} / \mathrm{dl} ; \mathrm{p}=0,026)($ Gráfico $\mathrm{l})$.

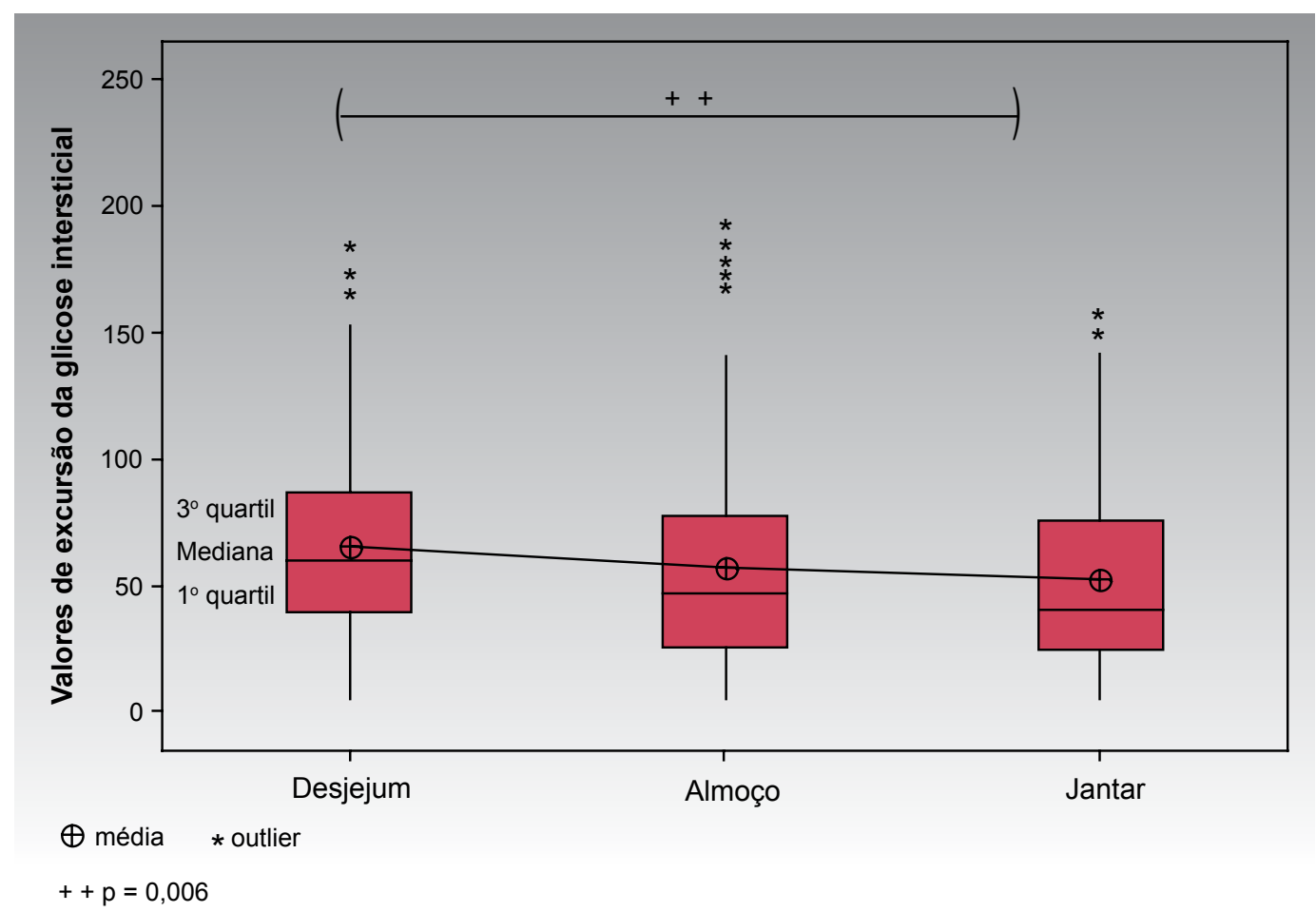

Gráfico 1. Excursões dos valores da glicose subcutânea nas principais refeições dos indivíduos com diabetes melito tipo 2 estudados. 
Avaliando cada par de variáveis, verificou-se que a excursão média no desjejum foi estatisticamente diferente da excursão média no jantar (95\%IC -24,96 a -1,66), o mesmo não acontecendo com os outros horários, ou seja, comparação entre desjejum e almoço (95\%IC -20,16 a $3,09)$ ou almoço e jantar (95\%IC -16,45 a 6,90).

O impacto do conteúdo de carboidratos na refeição foi avaliado em relação à excursão da glicose subcutânea. Demonstrou-se que esta relação possui diferenças significativas entre cada refeição $(\mathrm{p}=0,001)$ e que o aumento de glicose subcutânea por unidade de $\mathrm{CHO}$ é maior no desjejum $(2,142 \pm 2,203)$ que nas outras refeições (almo-

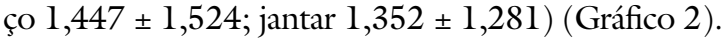

Dos indivíduos avaliados, 27 , ou $67,5 \%$, apresentaram picos de glicose subcutânea acima de $180 \mathrm{mg} / \mathrm{dl}$ uma hora após refeição, porquanto a maioria destes ( 14 ou $52,0 \%)$ no desjejum. Também ocorreram picos após o almoço (4 ou 15\%), simultaneamente no desjejum e no almoço ( 3 ou $7,5 \%)$, no jantar $(3$ ou $7,5 \%)$ e no lanche da tarde ( 2 ou $5 \%$ ).
Dos 13 indivíduos com HbAlc normal (até 6,0\%), sete ou $54 \%$ tiveram picos acima de $180 \mathrm{mg} / \mathrm{dl}$ uma hora após refeição. Entre estes sete pacientes, cinco deles faziam uso isolado de metformina.

\section{Correlação entre parâmetros de controle glicêmico com HbA1c}

As seguintes variáveis foram analisadas, conforme sua correlação com a HbAlc: GCJ, GCPD, GCPA, GCPJ, GCM (glicemia capilar média), glicose subcutânea média (GSM), valores de pico glicêmico capilar pós-prandial (PGCPP) e de glicose subcutânea pós-prandial (PGSPP) e excursões da glicose subcutânea no desjejum, almoço e jantar.

Não se encontrou correlação entre a HbAlc e a GCM, nem entre HbAlc e a GSM.

Encontrou-se correlação moderada entre a HbAlc e os valores de PGSPP (r 0,458; p 0,003) e de PGCPP ( $\mathrm{r} 0,418 ; \mathrm{p} 0,008)$. Houve correlação fraca entre a HbAlc e GCPA (r 0,205; p 0,03) e HbAlc e GCPJ

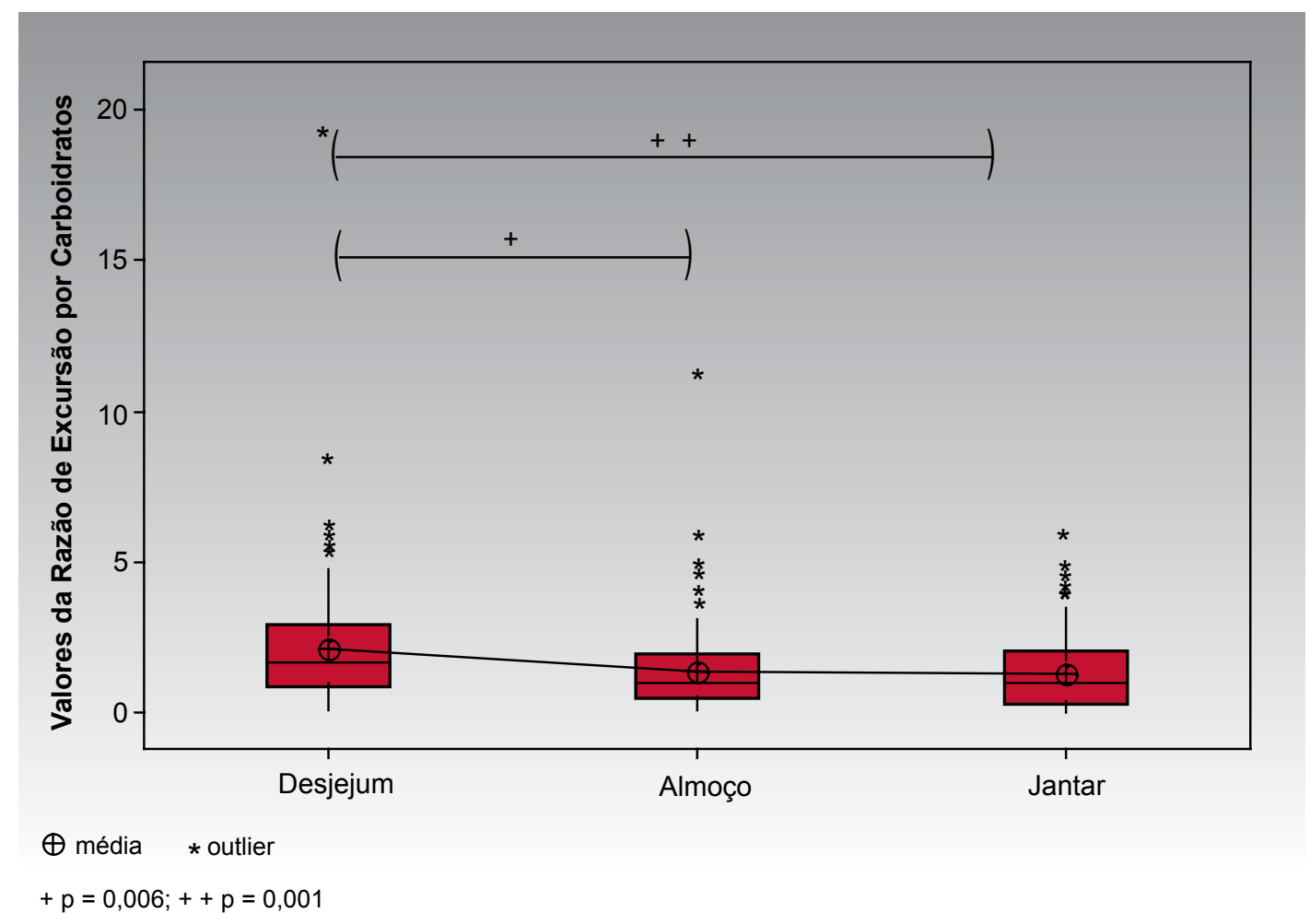

Gráfico 2. Valores da razão de excursão da glicose subcutânea pela quantidade de carboidratos ingeridos nos indivíduos com diabetes melito do tipo 2 estudados. 
(r 0,262; p 0,005), porém não houve correlação entre HbAlc e as excursões da glicose subcutânea (qualquer horário) e entre HbAlc e GCJ e GCPD.

\section{DISCUSSÃO}

A glicemia pós-prandial depende da interação de uma série de fatores. Embora a quantidade de carboidratos das refeições seja a principal determinante, outras variáveis intrínsecas e extrínsecas podem influenciá-la. As variáveis intrínsecas incluem o tipo específico de alimento ingerido, tipo de amido contido nele, estilo de preparação e grau de processamento. As variáveis extrínsecas incluem o nível de glicemia prévio à refeição, co-ingestão de outros macronutrientes (proteínas e gorduras), insulina disponível e resistência insulínica (20). O seu pico depende também do horário em que a refeição é realizada: sabe-se que após o desjejum, o pico é maior que em outros momentos do dia. Isso corresponderia à variação fisiológica, que possivelmente pudesse ser extrapolada aos portadores de diabetes melito (21).

No presente estudo, algumas particularidades da refeição do desjejum do nosso grupo de pacientes chamaram atenção: o predomínio do cardápio com base em "café e pão francês" foi nítido, com pequenas variações. Essa refeição composta basicamente de carboidratos, com baixa adição de outros macronutrientes e fibras (e, portanto, com alto IG), levaria à absorção mais rápida do carboidrato, ocasionando pico mais precoce e mais intenso. Estudos posteriores são necessários para confirmação dessa hipótese, bem como para definir se há também participação de um componente fisiológico inerente ao horário.

Em trabalho envolvendo 200 pacientes com DM2 em uso de glibenclamida e/ou metformina, valores glicêmicos mais elevados após desjejum em relação ao período após almoço foram observados por Monnier e cols. (22). Tal resultado seria explicado pela variação circadiana da produção hepática de glicose que ocorre em DM2, caracterizada pelo pico próximo ao amanhecer e declínio progressivo ao longo do dia (23). Em estudo nacional recente (24), realizado com glicemias plasmáticas, ficou evidenciado que este era o momento de maior excursão glicêmica, além de apresentar maior correlação com a HbAlc. Em nosso estudo, no entanto, não encontramos essa correlação. Também não houve correlação entre nenhum parâmetro de controle glicêmico com a HbAlc, mesmo quando foram testadas a GCM e a GSM. Achado, de certa forma, discor- dante da já conhecida excelente correlação existente entre a glicemia média de 7 pontos e a HbAlc, que varia de 0,81 a $0,95(25)$.

O fenômeno do amanhecer (dawn phenomenon), causado pela perda da sensibilidade insulínica hepática e extra-hepática induzida pela secreção noturna do hormônio do crescimento, também é outra possível explicação (26). Em artigo de revisão sobre esse tema, descreveu-se a prevalência deste fenômeno em torno de $55 \%$ dos pacientes com DMl e DM2 (27). Em pacientes com DMl em controle intensivo utilizando infusão contínua subcutânea de insulina (bomba de insulina), é descrita a freqüente necessidade de bólus de refeição maiores (em valores absolutos) e da necessidade de maior razão insulina/carboidrato no desjejum em relação às demais refeições (28).

Bonora cita observação freqüente (em torno de $70 \%$ ) de glicemias pós-prandiais altas (acima de 160 $\mathrm{mg} / \mathrm{dl}$ ) de 2 horas nas refeições de desjejum e almoço em pacientes com DM2 com HbAlc < 7\%, que não fazem uso de insulina (29). Dado relevante e que questiona o conceito de bom controle glicêmico, quando fundamentado no critério da HbAlc. Em nosso estudo, evidenciamos picos pós-prandiais $>180 \mathrm{mg} / \mathrm{dl}$ até mesmo em pacientes com HbAlc normal. O fato de cinco entre sete pacientes nessa condição estarem em uso de metformina em monoterapia poderia sugerir pouca eficácia do uso isolado dessa medicação.

A glicemia de jejum e a pós-prandial auxiliam no aumento da HbAlc, mas a contribuição relativa da hiperglicemia pós-prandial para as elevações da HbAlc é maior quanto mais baixo for o seu nível, conforme trabalho clássico de Monnier (30). O nosso grupo de pacientes é, por coincidência, muito parecido com o primeiro quintil de Monnier ( $\mathrm{HbAlc}<7,3 \%$ ), em que $70 \%$ do nível de HbAlc são explicados pela glicemia pós-prandial.

Em estudo recente (31), envolvendo 130 pacientes com DM2 avaliados por MGSC divididos em cinco grupos, de acordo com o nível de HbAlc (grupo 1 com HbAlc $<6,5 \%$ até grupo 5, com HbAlc > 9\%), os mesmos autores teorizam que a deterioração da homeostase glicêmica do DM2 evoluiria em processo envolvendo três etapas consecutivas: na primeira etapa ocorreria piora moderada da glicemia pós-prandial em todas as refeições, na segunda ocorreria piora acentuada no período da manhã (fenômeno do amanhecer) e na terceira, haveria hiperglicemia sustentada durante todo o período de jejum noturno. Nesse trabalho, a 
evolução da primeira para o segunda etapa foi demonstrada no grupo 2 (HbAlc entre $6,5 \%$ a $6,9 \%$ ) e no grupo 3 (HbAlc entre $7 \%$ e $7,9 \%$ ). Isso poderia também ajudar a explicar nossos achados, visto a semelhança do nosso grupo de pacientes com os primeiros três grupos do estudo citado.

No presente estudo não houve complicações decorrentes da utilização da MGSC, confirmando sua segurança. Alguns pacientes apresentaram dificuldades no manuseio do equipamento $(4 / 50$ ou $8 \%)$, que impossibilitaram a análise dos dados, o que indica que $o$ método foi bem tolerado. É inevitável observar que o alto custo desse exame o torna proibitivo para indicá-lo para a maioria dos pacientes com DM2 em nosso País. Contudo, conseguir-se-ia boa avaliação do controle glicêmico com o estímulo da automonitorização da glicemia capilar em vários momentos do dia, em todos pacientes quanto possíveis, e não apenas naqueles que apresentam evidências de controle insatisfatório.

No presente trabalho observou-se alta prevalência de picos de glicose subcutânea pós-prandiais (> 180 $\mathrm{mg} / \mathrm{dl}$, uma hora após refeição), até mesmo quando foram avaliados pacientes com HbAlc normal. A American Diabetes Association (ADA) recomenda que a mensuração da glicemia pós-prandial possa ser feita entre 1 e 2 horas, com nível-alvo menor que $180 \mathrm{mg} / \mathrm{dl}$, demonstrando preocupação em valorizar o momento do pico glicêmico (3).

Apesar de a predominância de picos após o desjejum, o achado da ocorrência em todas as refeições (inclusive lanche da tarde), reforça a necessidade da avaliação individualizada do paciente.

Então temos dois fatos: primeiro a ausência de correlação significativa entre as taxas subcutâneas e capilares de glicose e HbAlc. O segundo é a ocorrência de significativos picos de glicose subcutânea pós-prandiais ( 1 hora $>180 \mathrm{mg} / \mathrm{dl}$ ) em pacientes considerados como tendo controles bom e ótimo, segundo os critérios tradicionais. É razoável supor que os dois fatos estejam relacionados entre si e que juntos se expliquem.

Embora não se possa negar a valiosa importância já consagrada da HbAlc para avaliação do controle glicêmico, o presente estudo coloca mais um ponto de interrogação na utilização dela como único preditor de controle glicêmico, particularmente quando são considerados pacientes com DM2 com controle aceitável. A automonitorização da glicemia capilar em pacientes não tratados com insulina tem sido questionada, visto que o benefício encontrado na redução da HbAlc é pequeno quando este método é utilizado isoladamente (32-34), a ponto de a ADA considerar, em sua última recomendação, a sua utilidade nesta circunstância com grau de evidência E (35). Dessa forma, propõe-se que o modelo ideal de avaliação nesse subgrupo de pacientes seria a combinação do resultado da HbAlc com os dados da automonitorização da glicemia capilar pósprandial nos diferentes horários.

Como limitação deste estudo, pode-se citar a possibilidade de os pacientes terem se comportado de maneira diferente do habitual, pelo fato de saberem que estavam sendo monitorizados de modo contínuo. Isso poderia contribuir para a não correlação dos parâmetros glicêmicos com a HbAlc.

Concluindo, a análise das excursões pós-prandiais da glicose subcutânea por MGSC em 40 pacientes com DM2 bem controlados mostrou: (a) excursão da glicose subcutânea no desjejum maior que no jantar, além de elevação maior da glicose subcutânea por conteúdo de CHO consumidos no desjejum em relação ao almoço e jantar; (b) ausência de correlação entre as taxas de glicose subcutânea e glicemias capilares com HbAlc; (c) a MSCG foi eficiente em detectar picos pós-prandiais e ratificou a sua segurança e tolerabilidade.

O estudo também identificou alta prevalência de picos pós-prandiais de 1 hora, particularmente após o desjejum, inclusive em indivíduos com $\mathrm{HbAlc}$ normal.

Recomenda-se que na ausência da possibilidade de realização da MSCG, o incentivo à automonitorização da glicemia capilar pós-prandial nos diferentes horários deve ser feito em pacientes com DM2, otimizando o controle glicêmico em busca de metas mais estritas.

\section{AGRADECIMENTOS}

Agradecemos o apoio material da Abbott - divisão Diabetes Care e do Laboratório São Paulo (Araçatuba-SP, Dra. Lígia Avezum Galasini).

Agradecemos o apoio técnico de Francine Vilalta (análise estatística), Mieco Hashimoto (CGMS) e Sueli Contel Fortuna (revisão do abstract).

Não há potencial conflito de interesse que possa interferir na imparcialidade deste trabalho científico.

\section{REFERÊNCIAS}

1. The Diabetes Control and Complications Trial Research Group. The effect of intensive treatment of diabetes on the develop- 
ment and progression of long-term complications in insulindependent diabetes mellitus. N Eng J Med. 1993;329:977-86.

2. Stratton IM, Adler AI, Neil HA, Matthews DR, Manley SE, Cull $\mathrm{CA}$, et al. United Kingdom prospective diabetes study. BMJ. 2000;321:405-12.

3. American Diabetes Association. Standards of medical care in diabetes - 2006. Diabetes Care. 2006;29/1:4-42.

4. Home $\mathrm{P}$, Colagiuri S. A global guideline of type 2 diabetes: using a new "levels of care" approach. Diabetes Voice. 2005;50(4):22-4.

5. Tominaga $M$, Eguchi $H$, Manaka $H$, Igarasashi $K$, Kato T, Seikikawa A. Impaired glucose tolerance is a risk factor for cardiovascular disease, but not impaired fasting glucose. The Funagata diabetes study. Diabetes Care. 1999;22:920-4.

6. Rodrigues BL, Lau N, Burchfield CM, Abott RD, Sharp DS, Yano LK, et al. Glucose intolerance and 23-year risk of coronary heart disease and total mortality. The Honolulu heart program. Diabetes Care. 1999;22:1262-5.

7. The Decode Study Group, on behalf of the European Diabetes Epidemiology Group. Glucose tolerance and mortality: comparison of WHO and American Diabetes Association diagnostic criteria. Lancet 1999;354:617-21.

8. Malmberg $\mathrm{K}$, for the Digami study group. Prospective randomized study of intensive insulin treatment on long-term survival after acute myocardial infarction in patients with diabetes mellitus. BMJ. 1997;314:1512-5.

9. Shichiri M, Kishikawa H, Ohkubo $Y$, Wake N. Long term results of the Kumamoto study on optimal diabetes control in type 2 diabetic patients. Diabetes Care. 2000;23(2):B21-9.

10. Hanefel M, Cagatay M, Petrowitsh T, Neuser D, Petzinna D, Rupp M. Acarbose reduces the risk for myocardial infarction in type 2 diabetic patient: Meta-analysis of seven long-term studies. Eur Heart J. 2004;25:10-6.

11. Del Prato S. In search of normoglycemia in diabetes: controlling postprandial glucose. Intern J Obes. 2002;26(1):S9-17.

12. Heine RJ, Balkau B, Ceriello A, Del Prato S, Horton ES, Taskinen MR. What does postprandial hyperglycemia mean? Diabetic Med 2004;21:208-13.

13. Davies MJ. Post-prandial hyperglycemia and prevention of cardiovascular disease. Diabetic Med. 2005;22(1):6-9.

14. Bode BW, Schwartz S, Stubbs HA, Block JE. Glycemic characteristics in continuously monitored patients with type I and type II diabetes. Diabetes Care. 2005;28:2361-66.

15. Mastrolaro J, Levy R, Georges L-P, White N, Mestman J. Clinical results from a continuous glucose sensor multi-center study. Diabetes. 1998;47(1):A61.

16. Mastrolaro J. The minimed continuous glucose monitoring system (CGMS). J Pediatr Endocrinol Metab. 1999;12:751-8.

17. Bode, BW. Clinical utility of the continuous glucose monitoring system. Diabetes Technol Ther. 2000;2(1)S35-41.

18. Calles-Escandon J, Jaspan J, Robbins DC. Post-prandial oscillatory patterns of blood glucose and insulin in NIDDM. Abnormal diurnal insulin secretion patterns and glucose homeostasis independent of obesity. Diabetes Care. 1989;12:709-14.

19. Foster-Powell K, Holt SHA, Brand-Miller JC. International table of glycemic index and load index values: 2002. Am J Clin Nutr. 2002;76:5-56.

20. American Diabetes Association (ADA). Nutrition Recommendations and Interventions for Diabetes-2006. Diabetes Care. 2006;29:2140-57.
21. Gross JL, Ferreira SRG, Oliveira JE. Glicemia pós-prandial. Arq Bras Endocrinol Metab. 2003;47/6:728-38.

22. Monnier L, Colette C, Rabasa-Lhoret R, Lapinsk H, Caubel C, Avignon $A$, et al. Morning hyperglycemic excursions: a constant failure in the metabolic control of non insulin-using patients with type 2 diabetes. Diabetes Care. 2002;25:737-41.

23. Boden G, Chen X, Urbain JL. Evidence for a circadian rhythm of insulin sensitivity in patients with NIDDM caused by cyclic changes in hepatic glucose production. Diabetes. 1996;54:1044-50

24. Sartori MS, Aragon FF, Padovan CR, Pimenta WP. Contribuição da glicemia pós-desjejum para o controle glicêmico do paciente com diabetes melito tipo 2. Arq Bras Endocrinol Metab. 2006;50/1:53-9.

25. Rohlfing CL, Wiedmeyer HM, Little RR, England JD, Tennill A, Goldstein D. Defining the relationship between plasma glucose and HbA1c. Diabetes Care. 2002;25:275-8

26. Bolli GB. The dawn phenomenon: its origin and contribution to early morning hyperglycemia in diabetes mellitus. Diabetes Metab (Paris). 1998;14:675-86.

27. Carroll MF, Schade DS. The dawn phenomenon revisited: implications for diabetes therapy. Endocr Pract. 2005;11(1):55-64.

28. Brackenridge BP, Reed JH. Counting carbohydrates: the key to proper bolus. In: Fredrickson L, editor. The insulin pump therapy book. Los Angeles: MiniMed, Inc.; 1995.

29. Bonora E. Post-prandial peaks as a risk factor for cardiovascular disease: epidemiological perspectives. Int J Clin Pract Suppl. 2002;29:5-11.

30. Monnier L, Lapinski $H$, Colette $C$. Contributions of fasting and post-prandial plasma glucose increments to the overall diurnal hyperglycemia in type II diabetic patients: variations with increasing levels of HbA1c. Diabetes Care. 2003;26:881-5.

31. Monnier L, Colette C, Dunseath GJ, Owens DR. The loss of post-prandial glycemic control precedes stepwise deterioration of fasting with worsening diabetes. Diabetes Care. 2007;30(2):263-9.

32. Coster S, Gulliford MC, Seed PT, Powrie JK, Swaminathan R. Self-monitoring in type 2 diabetes mellitus: a meta-analysis. Diabetic Med. 2000;17(11):755-61.

33. Allen BT, DeLong ER, Feussner JR. Impact of glucose selfmonitoring on non-insulin-treated patients with type II diabetes mellitus. Randomized controlled trial comparing blood and urine testing. Diabetes Care. 1990;13(10):1044-50.

34. Farmer A, Wade A, Goyder E, Yudkin P, French D, Craven A, et al. Impact of self monitoring of blood glucose in the management of patients with non-insulin treated diabetes: open parallel group randomized trial. BMJ. 2007;335(7611):132.

35. American Diabetes Association. Standards of medical care in diabetes - 2007. Diabetes Care. 2007;30:S4-41.

Endereço para correspondência:

André Luiz Cruzes

Travessa Padre Feijó, 72

16200-093 Birigui, SP

E-mail: alcruzes@uol.com.br 